\title{
Observation of High-Field-Side Crash and Heat Transfer during Sawtooth Oscillation in Magnetically Confined Plasmas
}

\author{
H. K. Park, ${ }^{1}$ N. C. Luhmann, Jr., ${ }^{2}$ A. J. H. Donné, ${ }^{3}$ I. G. J. Classen, ${ }^{3}$ C. W. Domier, ${ }^{2}$ E. Mazzucato, ${ }^{1}$ T. Munsat, ${ }^{4}$ \\ M. J. van de Pol, ${ }^{3}$ Z. Xia, ${ }^{2}$ and TEXTOR team ${ }^{5}$ \\ ${ }^{1}$ Princeton Plasma Physics Laboratory, Princeton, New Jersey, USA \\ ${ }^{2}$ University of California at Davis, Davis, California, USA \\ ${ }^{3}$ FOM-Institute for Plasma Physics Rijnhuizen, ${ }^{*}$ Association EURATOM-FOM, P.O. Box 1207, 3430 BE Nieuwegein, The Netherlands \\ ${ }^{4}$ University of Colorado at Boulder, Boulder, Colorado, USA \\ ${ }^{5}$ Forschungszentrum Jülich GmbH*, Institut für Plasmaphysik, Association EURATOM-FZJ, D-52425 Jülich, Germany
}

(Received 6 December 2005; published 19 May 2006)

\begin{abstract}
High resolution (temporal and spatial), two-dimensional images of electron temperature fluctuations during sawtooth oscillations were employed to study the crash process and heat transfer in magnetically confined toroidal plasmas. The combination of kink and local pressure driven instabilities leads to a small poloidally localized puncture in the magnetic surface at both the low and the high field sides of the poloidal plane. This observation closely resembles the "fingering event" of the ballooning mode model with the high- $m$ mode only predicted at the low field side.
\end{abstract}

DOI: 10.1103/PhysRevLett.96.195003

Topological changes of the magnetic field configuration in plasmas, i.e., the magnetic reconnection process, have been observed in laboratory plasmas [1], solar flares [2], and interstellar space [3]. This Letter concerns the sawtooth oscillation [4], which is a repetitive benign disruptive behavior in which a nested magnetic field ruptures and stored plasma energy abruptly bursts out to the periphery. Numerous physical models have been developed to explain the changes in the magnetic topology, most of which have focused on developing an understanding from the first principles of the underlying magnetic reconnection process. In particular, the recent observation of sawtoothing radio frequency emissions from solar flares [5] suggests that the physical mechanism underlying this behavior may be universal in current carrying toroidal plasmas. Consequently, understanding of the sawtooth oscillation of current driven toroidal plasmas will be highly beneficial for solar and interstellar physics as well as for optimizing the control of magnetic fusion devices.

The sawtooth oscillation, known as the repetitive growth and decay of the core plasma pressure (and/or core current) through a magnetic reconnection process, in toroidal high temperature plasmas was discovered in 1974 [4]. An excellent review is available in Ref. [6]. Among the prominent models, the full reconnection model [7] suggests a complete exhaust of plasma pressure and current density through the reconnection process along the entire toroidal direction initiated by the pressure driven instability of the $m / n=1 / 1$ mode, where the plasma pressure ( $p=$ $\sum_{j=i, e} n_{j} T_{j}$ ) consists of particles (electrons and ions) at their corresponding temperatures and $m$ and $n$ are poloidal and toroidal mode numbers, respectively. The characteristic time scale is referred to as the reconnection time $\left(\tau_{c} \approx \frac{1}{2} \sqrt{\tau_{A}^{*} \cdot \tau_{\eta}}\right)$, where $\tau_{A}^{*}$ is the modified Alfvén transit time and $\tau_{\eta}$ is the resistive diffusion time and the typical
PACS numbers: 52.35.Vd, 52.55.- $\mathrm{s}$

value of $\tau_{c}$ is $\sim 700 \mu \mathrm{s}$ for the plasma parameters used in the experiment reported herein. Another model is the "quasi-interchange" model [8], where the cooler plasma convectively flows into the plasma core and the hot spot evolves into a crescent shape due to a strong distortion of the magnetic surface of the $m / n=1 / 1$ mode that results from the lack of magnetic shear in the core of the plasma. Reconstruction of the contours of constant soft x-ray emission on the Joint European Tokamak confirmed this model $[9,10]$. However, experimental verification of such a distinctively different evolution of the hot spot or island between the full reconnection model and the quasiinterchange model has been challenged by more sophisticated multiview $\mathrm{x}$-ray tomography $[11,12]$ and concluded that a unique solution of the inversion process based relatively small number of chords and views is not feasible. In addition, in current density profile measurements [13,14], only slight changes of current density were observed, while the reconnection time was much faster than expected on theoretical grounds. This discrepancy stimulated further modeling of the reconnection process. Observation of a localized electron temperature bulge $[15,16]$ during the sawtooth crash motivated researchers to introduce a model based on a finite pressure effect on the $m / n=1 / 1$ mode [17] at the "bad curvature" side of the magnetic surface (low field side) and a pressure driven ballooning instability $[18,19]$ combined with global stochasticity [17-19] of the field lines to explain the observed small change of the core current density in finite beta plasmas, where beta is the ratio between the plasma pressure $(p)$ and the magnetic energy $\left(B^{2} / 2 \mu_{0}\right)$. In moderate beta plasmas, such as those corresponding to the experimental results discussed here, the level of the ballooning instability and global stochasticity of magnetic field lines strongly coupled to the pressure surfaces is expected to be moderate compared to those in high beta plasmas. 
In order to reconcile the discrepancies between experiments and theoretical models and to improve the present theoretical understanding of the fundamentals of the reconnection process, one requires a direct multidimensional measurement of local plasma parameters, such as the local electron temperature and/or magnetic field in the core of the plasmas. Fortunately, such information has recently become available through the use of a two-dimensional (2D) electron cyclotron emission imaging (ECEI) system $[20,21]$ developed for the toroidal experiment for technology oriented research (TEXTOR) tokamak plasma. A vertically distributed array of antennas which images the plasma layer with an optical system similar to that of a camera with zoom lens is placed behind the imaging plane as shown in Fig. 3 in Ref. [21]; thus, radial resolution is maintained from the ECE frequency discrimination while vertical resolution is provided by the imaging optics. The sampling area of the image in TEXTOR at the focal plane is $16 \mathrm{~cm}$ (vertical) $\times 8 \mathrm{~cm}$ (radial) as shown in Fig. 1; the vertical resolution is determined by the optical element and is $\sim 2 \mathrm{~cm}$ for each pixel, and the radial resolution is $\sim 1 \mathrm{~cm}$ across the core of the tokamak plasma (total $16 \times 8=128$ channels). The time resolution is limited primarily by the digitizer, and the fastest time scale can be up to $5 \mu \mathrm{s}$. In the course of this experiment, many different sampling views were scanned in order to verify the extend of crash phenomena from the low-field-side mixing zone (up to $q \sim 2$ layer) to the high-field-side $q \sim$

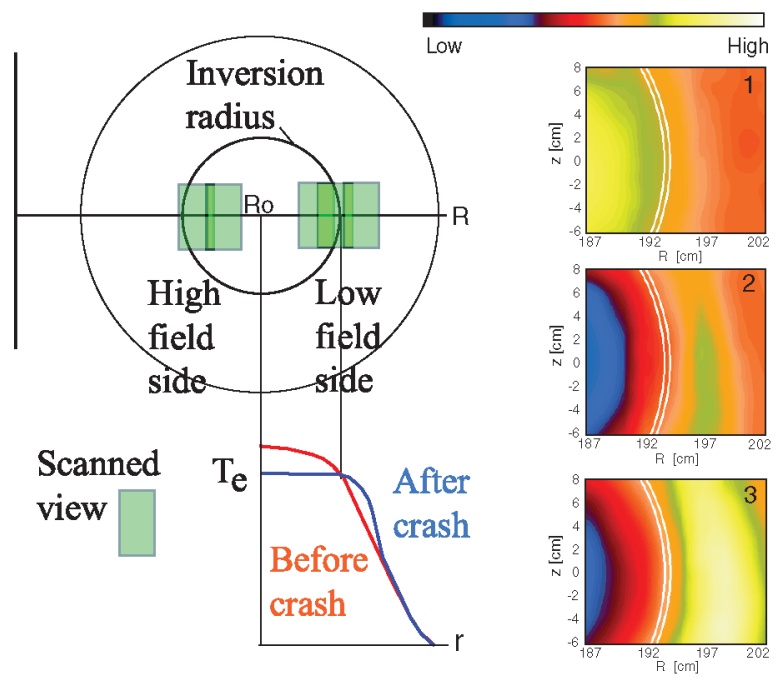

FIG. 1 (color). Scanned sampling views are shown with respect to the TEXTOR plasma during the course of this experiment; the range of the sampling view is from the $q \sim 2$ layer at the low field side to the $q \sim 1$ layer at the high field side. Based on time-averaged images from similar crash phases, composite images are constructed as shown in this figure; (1) heat is inside the inversion radius before the crash; (2) a hint of the heat is shown (greenish color) at the outside of the inversion radius, while the growth of the island (blackish blue color) is evident in the intermediate stage of the crash; (3) heat is accumulated outside the inversion radius after the crash.
1 layer as illustrated in this figure. The estimated inversion radius (white lines) is determined solely from the temperature fluctuation data, using the property that the relative variation of the temperature fluctuation is minimum near the inversion radius. Also, equilibrium analysis of the plasmas with similar plasma parameters indicates that $q \sim$ 1 is close to the inversion radius determined by this criterion. The fluctuation quantities are relatively calibrated to the averaged value obtained with a long integration time, and the intensity of the images is represented by $\delta T_{e} /\left\langle T_{e}\right\rangle$, where $T_{e}$ is the electron temperature, \langle\rangle is the time average, $\delta T_{e}$ is the fluctuation level $\left(=T_{e}-\left\langle T_{e}\right\rangle\right)$, and $\left\langle T_{e}\right\rangle$ is constant for the duration of many sawtooth oscillations. As demonstrated in a previous study of sawtooth physics by 1D ECE [15], the plotting of only the fluctuating temperatures (and subtracting the nonchanging, time-averaged temperature) simplifies the evaluation of the heat flow during the crash. The TEXTOR tokamak plasma has a circular shape with a major radius of $175 \mathrm{~cm}$ and minor radius of $46 \mathrm{~cm}$. The range of toroidal magnetic field in the present work was 1.9-2.4 $\mathrm{T}$, and the corresponding plasma current was $<305 \mathrm{kA}$. The $\mathrm{H}^{+}$plasma is heated with energetic neutral beams $\left(D_{0}, \sim 50 \mathrm{keV}\right.$, up to $\left.3 \mathrm{MW}\right)$ in order to maximize the temperature fluctuation of the sawtooth oscillation as well as to control plasma rotation (by varying the ratio of co- to counterinjection with respect to the direction of plasma current). The key plasma parameters were as follows: The central electron density and temperature range from 1.5 to $2.5 \times 10^{19} \mathrm{~m}^{-3}$ and from 1.2 to $1.6 \mathrm{keV}$, respectively. The corresponding peak toroidal beta is $\sim 1.0 \%$, and the average poloidal beta is between 0.3 and 0.5 . The toroidal rotation of the plasma varied from $\sim 1 \times$ $10^{4}$ to $\sim 8 \times 10^{4} \mathrm{~m} / \mathrm{s}$. The speed of a thermal electron is $\sim 6 \times 10^{7} \mathrm{~m} / \mathrm{s}$. The Alfvén and ion acoustic speeds are $5 \times 10^{5}$ and $7 \times 10^{5} \mathrm{~m} / \mathrm{s}$, respectively.

There are many sawtooth oscillations in a single discharge (one sawtooth period is $\sim 15 \mathrm{~ms}$, whereas the entire discharge duration is $\sim 5 \mathrm{~s}$ ): Each particular crash event may or may not occur within the field of view of the ECEI instrument, due to the random toroidal or poloidal phase of the localized crash region along the helical field structure. A representative sequence of $2 \mathrm{D}$ images illustrating the full view of the crash process observed at the low field side is shown in Fig. 2. This evaluation has nothing to do with the reproducibility of the crash process but, rather, concerns the fact that the phase of each crash event at the view position is dictated primarily by the timing of the local nature of the crash process and the toroidal rotation of the entire plasma. The hot spot, partially shown in whitish green color (frame 1), corresponds to a peaked electron temperature profile within the inversion radius (indicated as a black line). The temperature perturbation in frame 1 is still rather symmetric, but in later frames a clear distortion of the hot spot at the low field side becomes apparent. The growth of a sharp temperature point shown in frames 3 and 4 , which even crosses the inversion radius, is reminiscent of the theoretical reconnection process based on the "pres- 

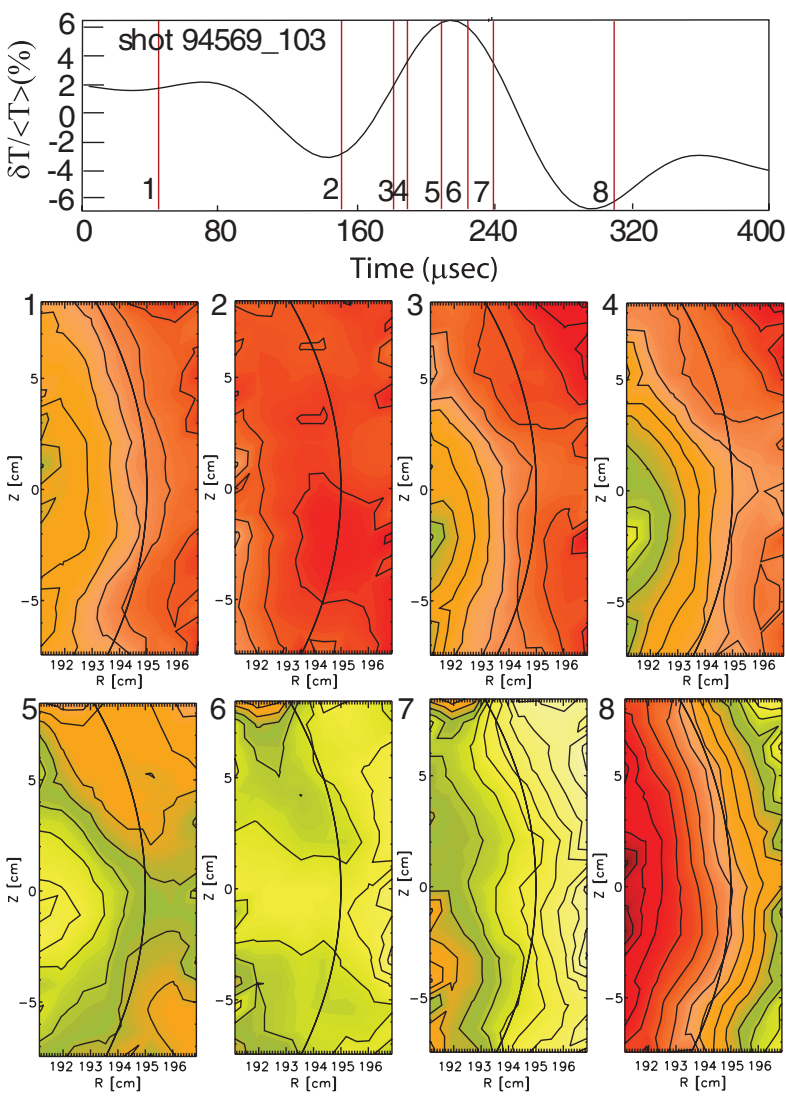

FIG. 2 (color). 2D images of the sawtooth crash at the low field side correspond to the times indicated in the electron temperature time trace at $z=0 \mathrm{~cm}, R=191 \mathrm{~cm}$. As the hot spot swells as shown in frames 3 and 4, a sharp temperature point is growing and crosses beyond the inversion radius. Eventually, the temperature point leads to the reconnection. Initially, it forms an "X point" in the poloidal plane (frame 5), and heat starts to flow to the outside through a small opening. The initial heat flow is highly collective, and the opening is increasing up to $\sim 15 \mathrm{~cm}$. At the end, the heat is accumulated outside the inversion radius, and the poloidal symmetry is recovered.

sure finger"'-ballooning mode discussed in Refs. [18,19]. From the images in Fig. 2, it appears that the heat punches through the "pinhole," and the crash zone grows as the heat transport is increased. The size of the crash zone increases to $\sim 15 \mathrm{~cm}$, and the heat transported outside the inversion radius is distributed along the poloidal and toroidal planes in a coherent manner. Since the time scale with the electron thermal velocity is on the order of nanoseconds and the progression of the images spans tens of microseconds, it is reasonable to assume that the transient heat flow follows the local magnetic field lines. It appears that the initial flow of heat is highly collective along the field lines. The spatial broadening of the heat outside the inversion radius shown in frames 6 and 7 can account for the toroidal spread of the heat, since the time it takes for electrons to complete one transit of the torus is on the order of a microsecond. At the end of the crash phase, the removed heat from the core is symmetrically accumulated in the mixing zone, and the temperature perturbation recovers symmetry as shown in frame 8 . It should be noted here that, although it is the absolute temperature that is related to the magnetic field topology, the temperature fluctuation contour plots and the associated heat flow are also a good indication of an opening in the magnetic surfaces.

In a second experimental campaign, the sample volume of the imaging system was moved to the "good curvature" side of the magnetic surface (high field side) in order to explore crash phenomena there. Theoretical models and experimental observations thus far suggest that the pressure driven ballooning mode instability is likely to grow in the regions of unfavorable curvature (low field side of the inversion radius) to release energy during the crash phase. Again, several tens of images of the sawtooth oscillations were scrutinized due to the local nature of the crash process and plasma rotation. It was found that the crash can occur at the high field side with the entire process quite similar to that at the low field side. The progressive detailed images of the crash process at the high field side are illustrated in Fig. 3. In the early stages, the hot spot is symmetric as shown in frame 1. In frame 4, a distortion of the hot spot is visible, and a sharp temperature point is formed but does not lead to a crash. As the pointed temperature surface is retreating, moderate swelling of the temperature (which is indicative of a growing kink instability or finite pressure effect on the $m / n=1 / 1$ mode) is shown in frame 5. In frame 7 , a larger sharp temperature point with a strong swelling of the hot spot (strong kink) pushes the sharp temperature point beyond the inversion radius, and heat starts flowing out in a collective fashion once the surface is opened. The first, but not complete, onset of a crash (frame 4), where a sharp temperature point is formed but does not lead to a crash, could be attributed to a lack of global pressure (energy from the kink or pressure bulge). However, the second attempt (frame 7) succeeds in opening the magnetic field, and the dimension of the opening starts with a small hole and grows up to $\sim 10 \mathrm{~cm}$, which is similar to the behavior in the low field side. As the heat is flowing out, the nested field lines from the core (cold spot) push the remaining heat from the rear as illustrated in frames $8-11$. The time evolution of the hot spot and island formation resembles a flattening process of the pressure profile as proposed by the full reconnection model [7]. As the heat is removed from the core, a closed field line topology is established again, and the poloidal symmetry is recovered as shown in frame 12. The observed sharp temperature point again resembles the ballooning mode but should be inhibited at the high field side. The entire sequence of the heat flow pattern is quite consistent with that of the low field case.

Advances in 2D visualization diagnostic techniques provided new insights of the physics of the sawtooth oscillation in the presence of strong guiding field. Improved theoretical modeling influenced by these findings will enhance the fundamental understanding of the magnetic 


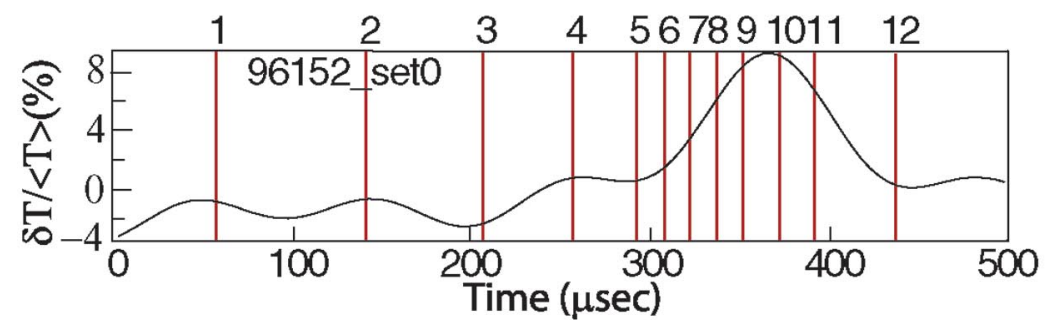

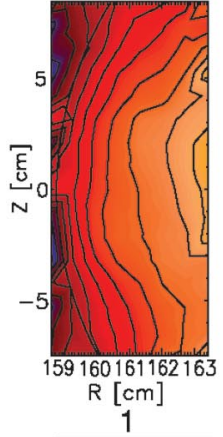
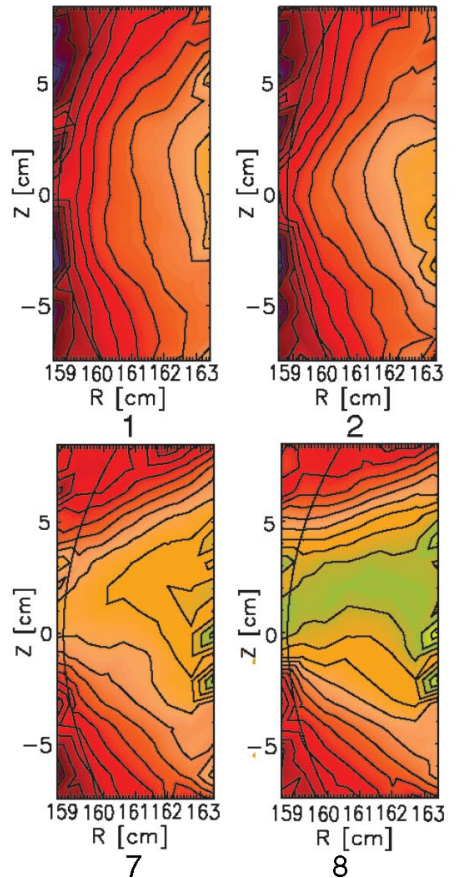
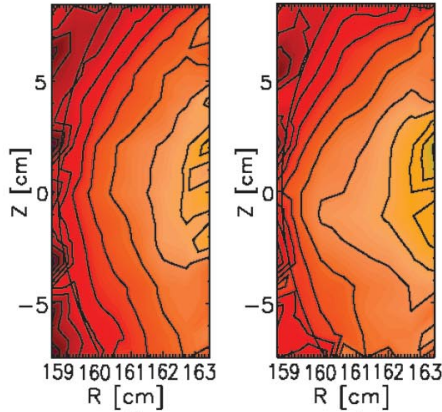

3

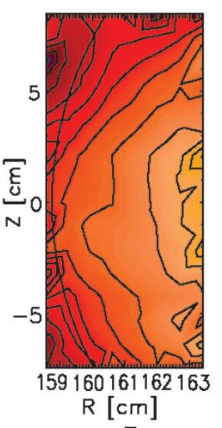

5
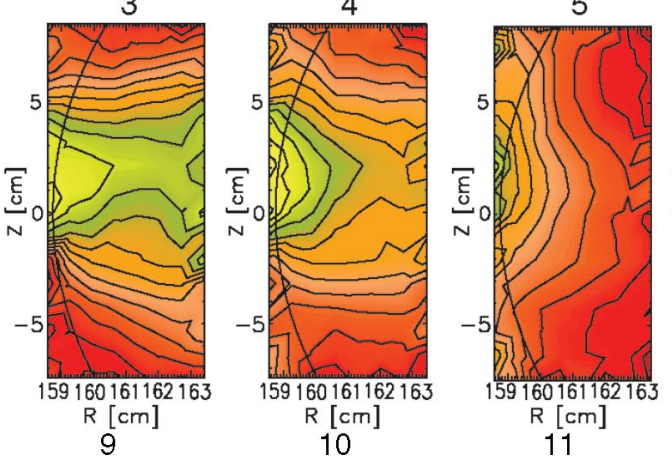

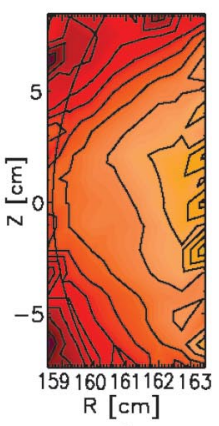

6

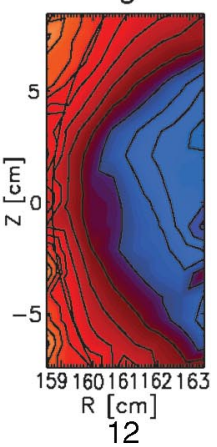

FIG. 3 (color). 2D images from the sawtooth crash at the high field side are shown with the time history of the electron temperature fluctuation at $z=0, R=148 \mathrm{~cm}$. The reconnection process is quite similar to that at the low field side. A sharp temperature point develops with the moderate swelling of the hot core (frame 4) but fails to lead to reconnection in the first attempt. In the second attempt, the sharp temperature point accompanied with the strong swelling of the hot spot (kink instability) (frames 6 and 7 ) succeeds in crossing the inversion radius through a small opening $(\sim$ a few $\mathrm{cm})$. The opening increases up to $\sim 10 \mathrm{~cm}$, and the heat flow is highly collective. The nested magnetic surfaces from the core push the heat flow out (frame 11), and, eventually, the symmetry is recovered (frame 12).

reconnection mechanism, which is beneficial for the control of future generations of magnetic confinement devices and may provide as well a better understanding of sawtooth phenomena in solar flares in which the guiding magnetic field is weak.

The authors are grateful to Dr. W. Park, Dr. E. Fredrickson, Dr. M. Yamada, Dr. E. Westerhof, and Dr. H. de Blank for valuable discussions. This work is supported by the U.S. DOE, Contracts No. DE-AC02-76CH0-3073, No. DE-FG03-95ER-54295, and No. W-7405ENG-48, NWO, and EURATOM.

*Partners in the Trilateral Euregio Cluster.

[1] M. Yamada, Earth Planets Space 53, 509 (2001).

[2] K. Shibata, Astrophys. Space Sci. 264, 129 (1998).

[3] D. Biskamp, Magnetic Reconnection in Plasmas (Cambridge University Press, Cambridge, England, 2000).
[4] S. von Goeler et al., Phys. Rev. Lett. 33, 1201 (1974).

[5] A. Klassen, Astron. Astrophys. 370, L41 (2001).

[6] R. J. Hastie, Astrophys. Space Sci. 256, 177 (1998).

[7] B. B. Kadomtsev, Sov. J. Plasma Phys. 1, 389 (1975).

[8] J.A. Wesson, Plasma Phys. Controlled Fusion 28, 243 (1986).

[9] A. E. Edwards et al., Phys. Rev. Lett. 57, 210 (1986).

[10] R. S. Granetz and P. Smeulders, Nucl. Fusion 28, 457 (1988).

[11] C. Janicki et al., Nucl. Fusion 30, 950 (1990).

[12] S. Yamaguchi et al., Plasma Phys. Controlled Fusion 46, 1163 (2004).

[13] H. Soltwisch, Rev. Sci. Instrum. 59, 1599 (1988).

[14] F. M. Levinton et al., Phys. Fluids B 5, 2554 (1993).

[15] Y. Nagayama et al., Phys. Plasmas 3, 1647 (1996).

[16] E. D. Fredrickson et al., Phys. Plasmas 7, 5051 (2000).

[17] W. Park et al., Phys. Fluids B 3, 507 (1991).

[18] W. Park et al., Phys. Rev. Lett. 75, 1763 (1995).

[19] Y. Nishimura et al., Phys. Plasmas 6, 4685 (1999).

[20] H. Park et al., Rev. Sci. Instrum. 74, 4239 (2003).

[21] H. Park et al., Rev. Sci. Instrum. 75, 3875 (2004). 